(C2007 IEEE. Personal use of this material is permitted. However, permission to reprint/republish this material for advertising or promotional purposes or for creating new collective works for resale or redistribution to servers or lists, or to reuse any copyrighted component of this work in other works must be obtained from the IEEE. 


\title{
Fuzzy Decision Support for Service Selection in E-Business Environments
}

\author{
Stefan Schmidt ${ }^{1}$, Elizabeth Chang ${ }^{2}$, Member, IEEE, Tharam Dillon ${ }^{3}$, Fellow, IEEE, and Robert Steele ${ }^{4}$, Member, IFEE \\ ${ }^{1,3,4}$ Stefan Schmidt, Tharam Dillon and Robert Steele, Faculty of IT, University of Technology, Sydney, Australia, \\ e-mail: (sschmidt, tharam, rsteele)@it.uts.edu.au \\ ${ }^{2}$ Elizabeth Chang, IT School of Information systems, Curtin University of Technology, Perth, Australia, \\ e-mail: elizabeth.chang@ebs.curtin.edu.au
}

\begin{abstract}
The emergence of semantic overlay networks as instruments to improve security, trust and stability in distributed virtual communities is recognized widely in the research community. We propose a fuzzy logic based framework which integrates social information such as trustworthiness, reputation and credibility ratings for individuals, alliances, organizations, services and products in e-commerce markets. This framework is designed to support the decision making process of autonomous agents during the selection of the optimal business partner. Fuzzy systems provide the ideal capabilities to process multiple criteria, which are composed of imprecise information and attribute definitions expressed in natural language. The proposed fuzzy models implement the DEco Arch framework and ontologies which provide details about concepts and their relationships in virtual communities.
\end{abstract}

Index Terms-Fuzzy Logic, Trust, Reputation, Credibility, Ontology, P2P, e-business

\section{INTRODUCTION}

The emergence of semantic overlay networks as instruments to improve security, trust and stability in distributed virtual communities is recognized widely in the research community [1-3]. Semantic overlay networks can provide social information such as trustworthiness, reputation and credibility ratings as well as risk evaluation ratings for individuals, alliances, organizations, services and products found in virtual communities. These different rankings can support the various decision making processes in digital ecosystems. However, current platforms and portals do not provide sufficient support for the processing of social information thus preventing the formation of socially strong communities. This is especially true in distributed e-business environments where we see a strong need for a more sophisticated integration of trust and reputation technologies [4]. Currently most e-commerce portals offer only simple ratings and reviews of products, services, resellers, service providers or manufacturers. These basic protection mechanisms are based on several weak assumptions:

The reviews and ratings are always truthful and not used to gain competitive advantages.

This assumption is unrealistic as monetary interests supersede honesty in many cases. For example the reseller of product X could rate its competitor negatively to overcome a disadvantage due to its higher price for product $X$. Other factors such as revenge or cultural and moral differences could also lead to inappropriate deviations from accurate ratings. Especially negative ratings can influence reputations and therefore significantly influence the success of participants in e-commerce portals [5]. As a result, it is up to the user to determine the credibility of the recommending peer in order to reach an unbiased conclusion.

Portal operators use appropriate calculation models for the aggregation of the provided social information.

Most portals do not provide sufficient transparency for users and expect users to trust the portal management with regards to calculation of social ratings, provision of all available information as well as the adherence to privacy policies. Many users accept these shortcomings as they trust the public reputation of major portals such as ebay.com or amazon.com. However, if the portal is not well known or new, or if the value of the exchanged goods or services is extraordinary high, then the user should not trust these portals by default and require better protection mechanisms.

All products and services are ranked in an unbiased and fair way - they may be ordered by price or rating.

This assumption is questionable as most commercial portals allow service provider to improve their visibility or the placing of their listing by monetary means. For example, a business may purchase a 'premium' package for its product listings within the portal which ensures that all listings are displayed on top of competitive product offerings even though their price is actually higher or their ranking is lower. Examples are Froogles' 'Sponsored Links' and Ebays' 'Featured Items'.

Next generation e-business portals and platforms will be hosted in truly decentralized peer to peer (P2P) environments where all data and information will be shared among peers instead of centralized portal servers. Users will employ intelligent, autonomous agents to discover and select appropriate products or services. Furthermore, these agents will negotiate contracts on behalf of their owners, monitor contract fulfilment and review the quality of service during or after the business transaction. All these activities are currently undertaken manually by users and prove to be very time consuming, ineffective and imprecise. Existing P2P technologies are able to provide all necessary components to realize this vision but users due to the lack of trust in autonomous e-commerce communities are currently unlikely to adopt such fully autonomous interactions. This lack of trust 
is mainly caused by inadequate social protection mechanisms. Once more transparent models which replicate human social relationships are integrated in e-business concepts the adoption by users is likely to increase.

In previous work, we have introduced the DEco Arch framework [6] which discusses the development of virtual communities in distributed digital ecosystems based on social ratings. Furthermore we have proposed detailed ontologies [7] which focuses on concepts for the integration of social relationships such as trust, reputation and credibility

In this paper we propose a fuzzy logic [8] based framework which implements the previously defined concepts and relationships. We identify and formalize specialized relationships between the interacting parties in e-business environments. For example, we identify the complex nature of the reputation concept which is influenced by several contextspecific criteria. In order to implement this concept we need a methodology that is not only capable of dealing with a varying number of attributes such as service, product or business alliance ratings but that also has the capability of processing dynamic attributes such as reputation trend or confidence values. Fuzzy systems provide the capabilities to process multiple criteria and attributes even if they are expressed in natural language [9]. Furthermore, Fuzzy logic based frameworks allow an intuitive and flexible approach for capturing vague information. Human beings often judge their surroundings based on vague information which is derived from past experiences, intuition and social relationships. Fuzzy systems allow us to incorporate such imprecise data into mathematical models to translate social values into crisp computer processible values. These crisp outputs can then be used to support autonomous decisions by the agents during the service or product discovery, selection, and QoS reviews [10].

This paper is organized in 5 sections. In section 2 we will discuss related work and the need for more sophisticated and adaptable integration of social networking into digital ecosystems. In section 3 we provide a brief introduction to the DEco Arch framework and its ontologies. In section 4 we propose a fuzzy logic based module which implements our ontologies in a powerful and flexible manner. Section 5 will then conclude this paper and provide a brief outlook for future work.

\section{RELATED WORK}

The provision of sophisticated and realistic decision support systems represents one of the most important challenges in current e-commerce environments. The fuzzy and dynamic nature of social values such as trust, reputation and credibility [11] present a key problem for the integration into both, centralized and distributed e-business environments. For example, Schweitzer et al. [12] discuss the fragile nature of trust and the negative impact of deception with regards to credibility, reputation and trustworthiness. Recovery from such trust violations is a slow and long process. In previous work we have proposed a fuzzy system based methodology for the discovery and handling of trust violations [13]. The trustworthiness of peers is often associated with its past behaviour which is constituted in its reputation rating. Another important factor for the evaluation of trustworthiness are opinions provided by trusted third party agents. Gu et al. [14] propose a generic decision support approach based on a fuzzy decision matrix. However, this work concentrates mostly on the integration of static attributes and does not provide sufficient integration of more dynamic concepts and attributes found in social overlay networks.

Ngai and Wat [15] propose an interesting approach to fuzzy system based risk and uncertainty evaluation. They identify different risk types and translate their findings into fuzzy sets [16] in order to generate composite risk rating. Risk is an important factor for context dependent decisions in social overlay networks. However, other, similarly important, factors such as trust, reputation, privacy, business need, price and other factors also need to be considered in order to support informed and confident decisions. Castelfranchi et al. [17] propose Fuzzy Cognitive Maps (FCM) in order to reach conclusions about the dynamic nature of trustworthiness and credibility. Lesani and Bagheri [18] analyse trust relationships in the semantic web and use trust graphs to infer about trust through associations among peers. However, similar to Castelfranchi et al. they do not take the composite nature of social ratings into account. Trust and reputation are not just simple or aggregated historic values [19] but rather a complex composition of feelings, experiences and strength and confidence values. For example, trust and reputation values for a product are often influenced by multiple criteria, such as the reputation of the manufacturer, the reputation of competitive products, the reputation of other businesses in the same context, seasonal differences, political criteria, etc [4].

\section{The DECO ARCH FramewORK AND ONTOLOGIES}

\section{DEco Arch Framework Overview}

The DEco Arch agent collaboration framework addresses current shortcomings [6] of service registration (and publication), service selection and service QoS ratings in ecommerce platforms and portals. The framework is hosted on a semantically enriched overlay network such as JXTA [20] which provides a virtual $\mathrm{P} 2 \mathrm{P}$ environment and, thus, overcomes the dependency on a centrally hosted e-commerce portal. Social information such as trustworthiness, reputation and credibility values are stored separately in a publicly accessible DHT [21] which offers further independence and fault tolerance. The DEco Arch framework not only provides individually accessible social information about all aspects of a business but also emphasizes the importance of group alliances. Group alliances are groups of collaborating agents, which offer complementary or similar services within the same context - similar to categories in a yellow pages directory. 


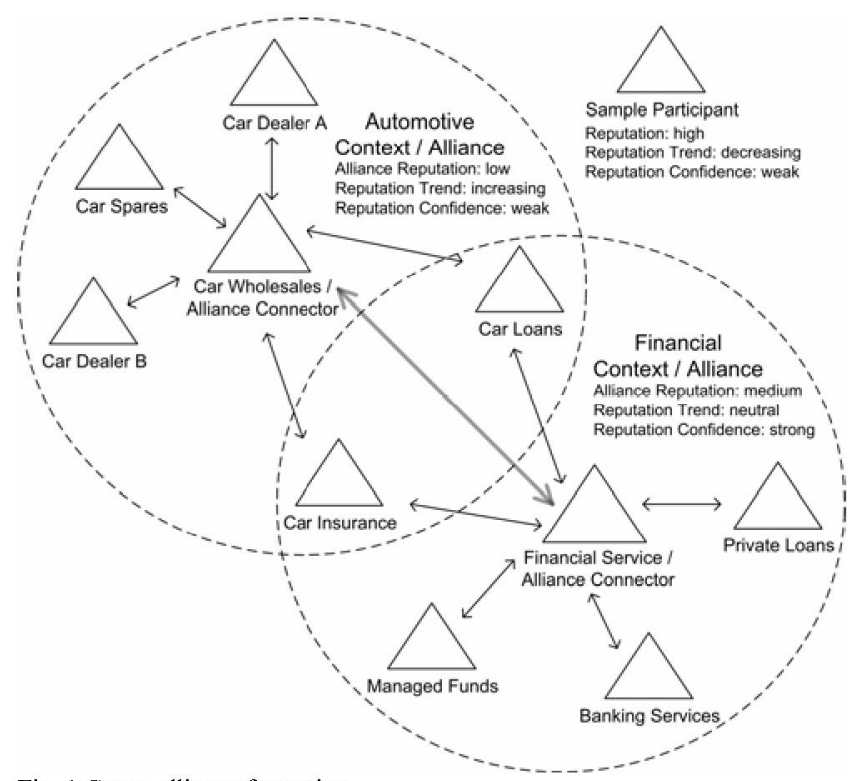

Fig. 1 Group alliance formation

All business agents and group alliances have distinct trust and reputation values assigned to them. These social ratings stabilize confidence within the network by excluding malicious peers. The introduction of reputation values for group alliances is founded on social observations where people have often prejudices towards certain contexts or categories such as car sales or insurance salesmen whereas other contexts enjoy a fairly high reputation such as community services or hospitality. Furthermore, low initial ratings of new group alliances or individual agents help to overcome common attacks in P2P networks such as self replication [22] or replay of ratings used by agents to gain an advantage over their competitors.

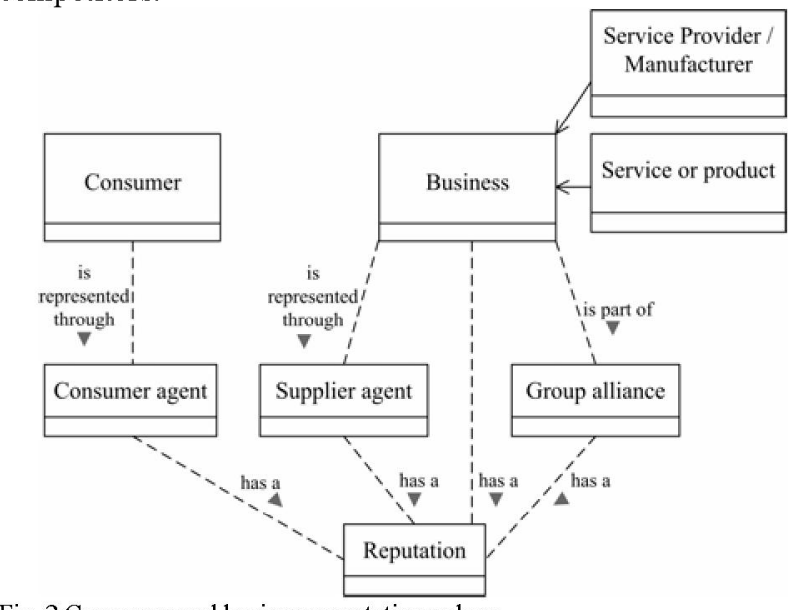

Fig. 2 Consumer and business reputation values

Reputation values for group alliances are not supplied by recommending agents but rather calculated as the weighted average of all reputation values of members of a group alliance. Reputation values of individual agents which contribute to the reputation value of a group alliance are weighted by a membership percentage value of each agent within an alliance. Social ratings are a decisive criterion during the service selection process undertaken by the consumer agent. Service provider agents, who want to register a service description, need to join one or more group alliances in order to be visible within the network. If an agent can not discover a suitable group alliance which matches its service description and context to a certain degree, it may establish a new alliance.

\section{DEco Arch Trust and Reputation Ontologies}

The DEco Arch ontologies [6] formalize and facilitate autonomous interactions between intelligent agents in centralized and decentralized e-business environments. The ontologies focus on the integration of social factors such as trustworthiness, reputation and credibility concepts during the formation and stabilization of unsupervised virtual communities. We provided detailed descriptions of concepts and their relationships with regards to essential problems such as business discovery, business selection (with and without recommendations from third party peers) and the review of the quality of service during and/or after the business interaction. These ontologies offer a common set of concepts and their relationships and reflect the complex nature of social network with specific focus on e-business. The adherence to such ontological concepts will improve interoperability between the various platforms and frameworks and, therefore, improve transparency, accessibility and increased confidence for all involved parties.

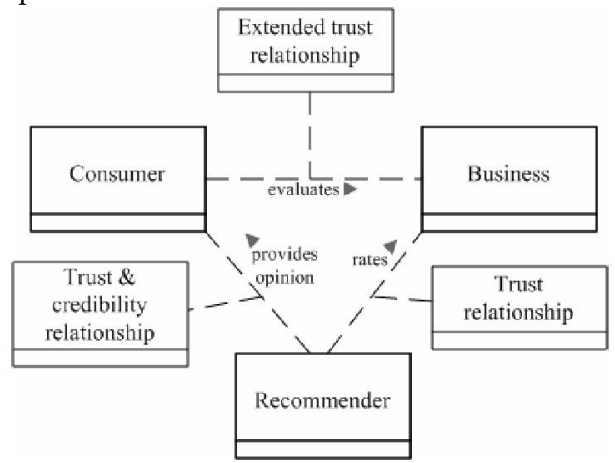

Fig. 3 General Social Relationships Ontology for Service Selection [7]

\section{Fuzzy Calculation Modules}

In our previous work we have discussed ontologies [7] which formalize the concepts and concept relationships used in the DEco Arch framework [6]. In order to translate these concepts and relationships into computational logic we need to introduce methodologies and implementations for the quantification of the social values such as trust, reputation and credibility. Cognitive science shows us that trust and reputation are of a fuzzy nature [11] and are, therefore, hard to quantify. Human beings base their trustworthiness, reputation and credibility measurements on vague feelings, past experiences and opinions from third parties. While humans express these feelings as linguistic variables which are 
individually defined, computational logic requires the translation of these variables into numeric ranges.

Fuzzy logic offers mathematical concepts that support flexible and customizable translations of fuzzy variables into the crisp outputs that computational logic requires. Fuzzy logic tolerates imprecise inputs and, hence, allows a certain level of uncertainty. Furthermore it allows individual setups according to specific user requirements. For example, some users may have little confidence in the abilities of intelligent agents which act on their behalf in next-generation autonomous ebusiness environments. To improve confidence into such autonomous frameworks two measures may be taken. Firstly, we must use mathematical approaches which are commonly understandable and based on natural language. Secondly, the user must be able to control the decision making capabilities of the agent based on his individual security requirements. Both measures are realized by fuzzy rule bases which offer maximum transparency to the user as they are defined by the user himself and are easy to use because the user uses linguistic terms to create individual rules.

These linguistic expressions allow individual settings at different levels of detail. The level of detail can be adjusted according to the context, risk or user group of the e-business environment. A large scale e-commerce transaction between multi national enterprises is likely to require very fine-grained security settings. On the other hand most e-business portals which target private customers and small businesses such as ebay.com or amazon.com need easily configurable or preconfigured trust and reputation systems which allow mass user adoption. Therefore the end user will be equipped by with predefined modules that are part of a fuzzy inference system such as fuzzy rule bases, fuzzy membership functions and defuzzification functions. Such modules are configured by domain experts. The portal operator may also offer preconfigured modules for different user types. If required, users can then extend or change these configurations to meet their individual security needs. For example, fuzzy rule bases offer sufficient flexibility to accommodate different levels of detail. Fuzzy rule bases could be composed of only 5 rules or 300 rules, depending on the desired level of control and existing expert knowledge of the end user [23]

In a scenario where an autonomous agent may have discovered a number of potential business partners which offer the desired products or services, the agent needs to make an informed decision in order to select the optimal business partner. Similarly, for high value transactions businesses or resellers need to evaluate the reputation, trustworthiness, business risk, or simply the business value of individual customers. In this section we first discuss an exemplary fuzzy system as used frequently in our framework and then introduce two procedural models which make use of fuzzy systems to facilitate autonomous selection and evaluation processes. We examine a consumer evaluation model for businesses and a second model for consumers to evaluate businesses. These models integrate several criteria such as social values, cost, business or consumer specific requirements, and consumer or business values.

\section{General Fuzzy System for Reputation Evaluation}

In the following we briefly demonstrate the generic design of a fuzzy controller (Fig. 4) for the reputation evaluation of entities such as products, services, manufacturers, resellers and alliances. The reputation rating of an entity will be computed from three factors which influence its final value. The first factor is a weighted average of all available reputation values for this specific entity in a given context and times slot. These are either taken from local records and are thus based on past business interaction information; or if this data is not existent or outdated, it is taken from the entity ratings from recommending agents. The classification we use for the reputation value is closely related to the work presented by Chang et al [24]. The reputation value is represented as a fuzzy set with five fuzzy variables namely, 'very bad reputation', 'little reputation', 'some reputation', 'good reputation', and 'very good reputation' where the Universe of Discourse (UoD) is between 0 and 5 as discussed in [24]. Opinions delivered by peer agents or ratings received from publicly accessible review platforms follow their own rating scales. We use a simple function to normalize and scale each reputation value $\left(r_{\text {val }}\right)$ from the range [reputation scale min $\left(r_{m i n}\right)$, reputation scale $\left.\max \left(r_{\max }\right)\right]$ to our reputation value range of $[0,5]$ as depicted in expression (1):

$$
r_{i}=\frac{r_{v a l}-r_{\min }}{r_{\max }-r_{\min }} * 5
$$

In a next step, we calculate the sum of all exponentially weighted time factors $w$. To achieve this, the collection of reputation values (with size $N$ ) is sorted and numbered (with the label $i$ ) by its timespots and then calculated as shown in in expression (2a) where $n$ denotes the current timespot, $m$ denotes the timespot of the reported reputation value. This aggregated weight factor is required in expression (2b) as a denominator during the calculation of individual weight values which, when aggregated, equal to 1 .

$$
\begin{aligned}
& w=\sum_{i=1}^{N} e^{-\frac{(n-m i)}{\left(n-m_{1}\right)^{*} D}} \\
& w_{i}=\frac{e^{-\frac{\left(n-m_{i}\right)}{\left(n-m_{1}\right)^{*} \alpha}}}{w} ; \text { where } \sum_{i=1}^{N} w_{i}=1
\end{aligned}
$$

Next, we calculate an exponentially weighted average value over all available reputation values. The reputation values are weighted by their timeliness within the time spot collection using expression (2c):

$$
r_{w}=\sum_{i=1}^{N}\left(w_{i} * r\right)_{i}
$$




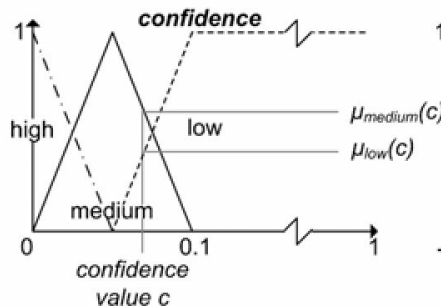

Step 2: inference process of fuzzified input variables and fuzzy rules

$$
\begin{aligned}
& \text { Fuzzy Rule Base: } \\
& \text { 1. IF } c \text { IS low AND } t \text { IS low AND } r \text { IS very bad } \\
& \text { THEN reputation IS very bad. } \\
& \text { 2. IF } c \text { IS low AND } t \text { IS increasing AND } r \text { IS very bad } \\
& \text { THEN reputation IS little. } \\
& \text { 3. IF } c \text { IS medium AND } t \text { IS neutral AND } r \text { IS little } \\
& \text { THEN reputation IS little. } \\
& \text { 27. IF } c \text { IS high AND } t \text { IS neutral AND } r \text { IS very good } \\
& \text { THEN reputation IS very good. }
\end{aligned}
$$

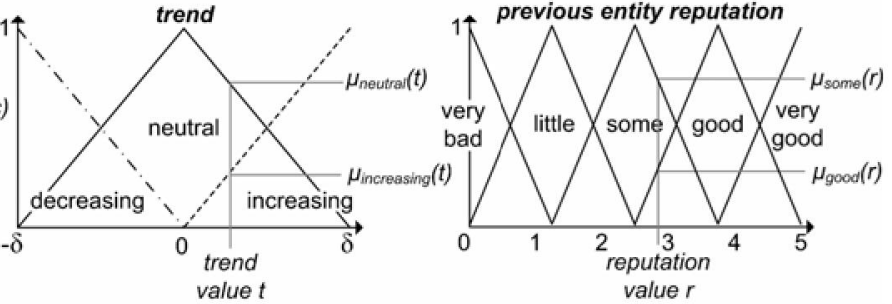

Step 3: defuzzification of output space into crisp reputation value

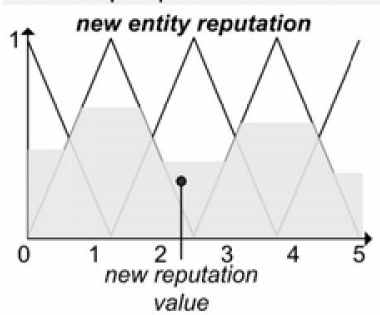

Fig. 4 Generic Mandami Fuzzy Inference Engine (3 input - 1 output)

We use an exponential function to model the reputation decay over the time horizon between the first reported reputation value $\left(m_{1}\right)$ and the current time $(n)$. The exponential function allows us to put more emphasis on current data by allocating higher weights to more recent reputation information. The time-based weighting of reputation values will, for instance, reduce the overall reputation value stronger (compared to a moving average) if the recent behaviour of the reputation queried agent has led to significant drops after malicious behaviour. Furthermore, our tests show that moving averages are not suitable for large datasets as they do not prioritise recent information over older datasets. The shape of the exponential function is adjustable by the denominator $\alpha$ which characterizes the rate of decay. For example, the function is highly curved when $\alpha$ is 0.3 and almost linear for a $\alpha$ of 2 . Users who wish to emphasize the calculations of their agent on more recent data rather than historic data can therefore adjust $\alpha$ to their preference.

The second influencing factor for the reputation rating is a trend value which describes the progress of the reputation value of the evaluated entity over time. With the help of this trend value it is possible to determine whether the reputation of an entity has been improving or deteriorating lately [6]. While all available historic reputation data is included in the calculation of the trend value, more recent information is provided with higher (exponentially) weighted factors. The reputation trend value $\left(t_{R}\right)$ is calculated with expression ( $3 a$ ). We determine the relative changes of the sorted reputation value collection and multiply these values with the exponential weights $w_{i}$. The calculation of the exponential weights is analogous to the calculation of the previously discussed weighted reputation value from expression (2b). The trend value is translated into a fuzzy set with three variables 'decreasing', 'neutral', and 'increasing' where the UoD is in the range between $-\delta$ and $\delta$. $\delta$ represents the maximum change between two adjacent reputation values plus an additional factor $\beta$ which extends the UoD of the trend variable. $\beta$ can be chosen from experience, or trial and error.

$$
\begin{aligned}
& t_{R}=\sum_{i=1}^{N}\left(w_{i} *\left(r_{w}-r_{w-1}\right)\right) \\
& \delta=\max \left(\left|r_{w}-r_{w-1}\right|\right)+\beta
\end{aligned}
$$

The final influence on the reputation value is the confidence value which quantifies the significance of the reputation value. We represent the confidence as the standard error of the mean $c_{R}$ (see expression 4). The more reputation data is available for the calculation of the overall reputation value the smaller is $c_{R}$. The smaller $c_{R}$ the higher our confidence in the reputation of an entity. More specifically, the size of $c_{R}$ is inversely proportional to the square root of the dataset size $N$.

$$
c_{R}=\frac{s_{R}}{\sqrt{N}}
$$

The confidence value is translated into a fuzzy set with three variables 'low confidence', 'medium confidence', and 'high confidence' where the UoD is in the range between 0 and 1. Our tests show that the standard error of the mean is not larger than $10 \%$ in most cases. The value ranges for the fuzzy sets are adjusted accordingly (see Fig. 4 Step 1). If no previous ratings or reputation records exist about a specific entity we assume that the entity is new in the community. The new entity will be assigned a start value of 0 which is the lowest possible rating in the given $\mathrm{UoD}$. The trend value will initially be set to increasing in order to give the newly registered entity a start value from which has a chance to improve. The startup confidence value will be set to 'low'. 
Stage 1:

Consumer Reputation Calculation
Stage 2:

Consumer Criteria Integration

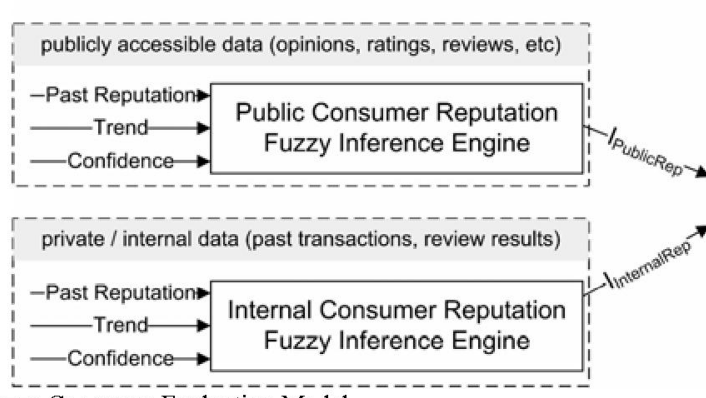

Fig. 5 2-Stage Fuzzy Consumer Evaluation Model

A fuzzy inference system is then designed as a classic 3 input (past reputation value, strength, and confidence) and 1 output (new entity reputation value) system with a user defined entity-dependent fuzzy rule base (Fig. 4). The creation of entity-specific rule bases allows the user to express his individual perception of security, trust and risk. For example, the user would like to purchase a digital camera. He specifically wants to buy a specific model by manufacturer $X$ but he is indifferent to the reseller or the platform on which the camera is bought. In this case the user would create a rather restrictive rule base to make sure that this specific requirement is met. In our model, he would thus attach great importance to product and manufacturer reputation values while he would place less emphasis on the influence of reseller reputation values.

The newly calculated reputation values for products/services, manufacturers/service providers, resellers, and group alliances are then fed into the business reputation inference engine in order to generate a composite trustworthiness value for the evaluated business. Furthermore, the reputation values, which are calculated from past business interaction records, are used as additional input for the business reputation inference engine. The engine then generates a composite reputation value for the evaluated business. In a final step, the business value factor is calculated by the business value fuzzy inference engine by taking the calculated reputation values for the evaluated entities as input along with the previously calculated service need correlation value and the cost of the product or service. The business value factor can now be used for direct comparisons between potential business partners. The trusting agent then selects the business with the highest value according to its individual business need, price and other criteria.

\section{Fuzzy Customer Evaluation Module}

Businesses which exist in an oligopolistic market where few sellers control a high percentage of total sales we need fast and efficient evaluation models to determine the business value of customers. These evaluations may then be used to offer special deals to long-term trustworthy customers, negotiate special contracts, or reject non-reputable customers due to high risks. The fuzzy customer evaluation module offers these evaluation capabilities and is composed of two stages. In stage 1 the business agent calculates a public and an internal reputation value for its customers by collecting publicly accessible reputation information about a consumer and aggregating these values through expression (2c). Furthermore, he determines the trend of this information according to expression (3) as a crisp number. Depending on the amount of available information the business agent is also able to determine its confidence in the existing aggregated rating values according to expression (4).

The reputation, trend and confidence values are then fuzzified as depicted in step 1 of Fig. 4. In a next step the fuzzified inputs are inferred with the predefined fuzzy rule base. In a Mamdani [25] model all firing rules are aggregated into an output fuzzy set which will then be defuzzified using the centre of gravity (COG) method. This crisp output value represents then the public reputation value for this customer. The same process is then repeated to calculate the internal reputation value for this customer which is based on data collected from direct interaction during past business interactions with this customer. If this data does not exist, the business agent will only use the public reputation value and proceed to stage 2 .

In stage two the business agent integrates these social values with other criteria such as pre-evaluated risk factors and the business need. The consumer risk factor is determined by the impact of potential loss or damage. These factors are then fed into the consumer value fuzzy inference engine in order to calculate a crisp output which indicates the overall value of a specific consumer for this business. This resulting consumer value factor is then useful during contract and price negotiations, targeted advertising programs or other incentive programs. For example, banks distributing credit cards need to evaluate the value of customers which may churn away soon. If the reputation and the business value of these customers is high, the bank may offer them financial incentives to prevent customer attrition. 
Stage 1

Stage 2:

Stage 3

Individual Entity Reputation Evaluation

Overall Bușiness Reputation Calculation Business Criteria Integration

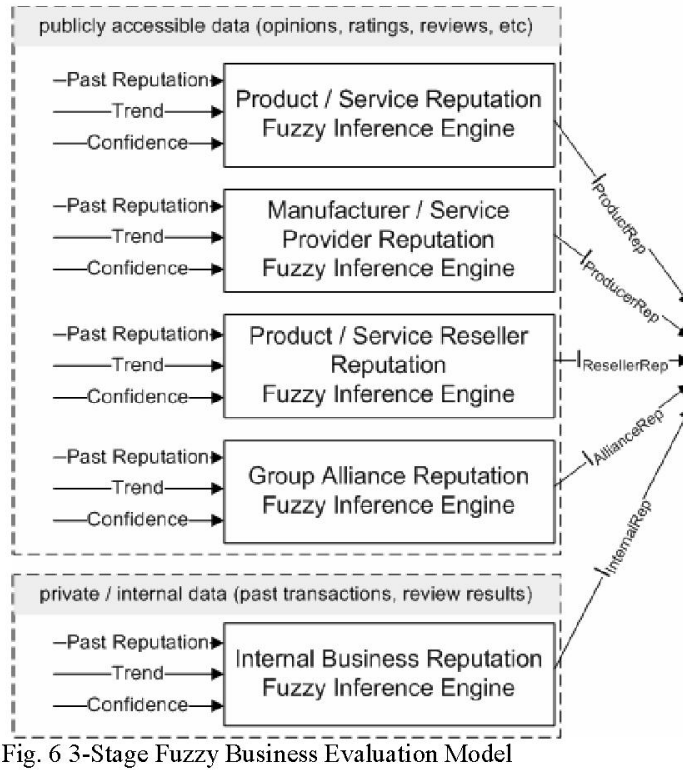

Fig. 63 -Stage Fuzzy Business Evaluation Model

\section{Fuzzy Business Selection Module}

A number of calculations are required to provide decision support during autonomous selection of the optimal business partner. For example, in the real world the consumer usually establishes the reputation values of several entities which ultimately influence the overall trustworthiness value for a business. These entities include the service or product reputation, the service provider or manufacturer reputation, the reputation of the reseller, and the reputation of the group alliance. The more of this reputation information is available to the consumer the better will be the precision of its calculations which will enhance user confidence. However, not all of these entities are always involved, for example, in some cases the manufacturer sells its products directly and therefore the reseller does not exist

Our model for the evaluation of businesses consumers is composed of three stages (see Fig. 6). Stage one provides four individual fuzzy systems for the evaluation of reputation values for products or services; manufacturers or service providers; product or service resellers; and group alliances. Furthermore, the consumer agent can calculate a reputation value for the evaluated business partner from its internal records of past interactions with this business partners in the same context. The fuzzy reputation evaluation systems in stage one are similar to the one described in the customer evaluation module where we have a aggregated reputation value, a trend value and a confidence value as inputs for the fuzzy inference system.

Every fuzzy inference system will generate a reputation output value which is then used as input value for the business reputation fuzzy inference engine as part of stage two in the business selection module. This fuzzy inference process will then generate an overall reputation value for the evaluated business

In stage three this overall reputation value for the business is used as input for the business value fuzzy inference engine which also integrates other criteria such as cost related criteria a product or service need criterion, a specification correlation criterion and a business risk criterion. As a result of the last stage of the fuzzy business selection module the consumer agent receives an overall business value factor. These business value factors are then used for direct comparisons between all potential business partners and the agent can select the business partner which fulfils all requirements in the most optimal way.

\section{Benefits of fuzzy decision support}

The provision of ratings or reviews about businesses and products or services in e-commerce environments is quite common. However, most of these rating systems are too simplistic and do not reflect the complex relationships among the interacting parties. There are often no incentives to encourage users to tell the truth due to the lack of credibility monitoring. Furthermore, most platforms do not provide trust or reputation ratings about customer and therefore provide no effective risk management capabilities for businesses. This is especially common for small scale e-commerce interactions. For high value enterprise level business interactions the provision of social factors such as trustworthiness or reputation for the assessment of consumers is even more important as the risks are increasing with the value of exchanged goods or 
services during the transaction.

Most of these platforms are designed for humans only and do not provide interfaces for autonomous agents. The DEco Arch framework provides an architecture which encourages the formation of virtual communities. Autonomous agents interact to discover and select businesses, to negotiate contracts, and agents communicate to exchange opinions about reputation and trustworthiness values derived from the review of contracts. In order to equip autonomous agents with all these capabilities we need to use mathematical concepts which resemble human thought processes and allow the translation of such vague and imprecise information into crisp values. Fuzzy logic offers these capabilities and furthermore allows the allocation different importance factors to the various input criteria in a flexible approach. For example, the owner of a consumer agent may want to attach more importance to the cost criterion than the business reputation.

\section{Conclusion}

In this paper we presented several fuzzy system modules which are designed to support autonomous decision making in virtual communities. These modules implement and support the DEco Arch framework [6] and its ontologies [7] which formalizes and facilitates autonomous interactions between intelligent agents in centralized and decentralized e-business environments. The introduced fuzzy system modules focus on the integration of social reputation and trustworthiness factors during the evaluation of the value of the business partner. Furthermore, the modules are able to integrate multiple criteria such as cost, risk, social criteria, and business related criteria in a flexible manner through customized fuzzy rule bases. We have organized our fuzzy customer and business evaluation models in separate stages. This facilitates customization and extensions to meet the specific requirements for the evaluation and selection processes of different users.

We have shown that fuzzy logic is ideal for the translation of imprecise information such as trustworthiness, reputation and credibility ratings into crisp values which are crucial for computational logic. Moreover, Fuzzy logic offers transparency to the user through the incorporation of natural language and easily comprehendible functions. In order to put emphasis on individual security, social, or other criteria, users with sufficient domain knowledge can customize or extend existing rule bases or inference systems without the need to learn complex mathematical concepts. Our implementation provides predefined fuzzy inference systems and rulebases to reduce initial setup requirements while still offering optional customization for advanced users. Due to space limitations of conference proceedings we present our test results on the eXel lab website [26].

\section{REFERENCES}

[1] C. Tang, Z. Xu, and S. Dwarkadas, "Peer-to-peer information retrieval using self-organizing semantic overlay networks," Proceedings of the
2003 conference on Applications, technologies, architectures, and protocols for computer communications, pp. 175-186, 2003.

[2] K. Aberer, P. Cudre-Mauroux, M. Hauswirth, and T. Van Pelt, "GridVine: Building Internet-Seale Semantic Overlay Networks," International Semantic Web Conference, pp. 107-121, 2004.

[3] A. Crespo and H. Garcia-Molina, "Semantic Overlay Networks for P2P Systems," Submitted for publication, 2004.

[4] S. Schmidt, R. Steele, T. Dillon, and E. Chang, "Fuzzy Trust Evaluation and Credibility Development in Multi-Agent Systems," Applied Soft Computing, 2006.

[5] S. S. Standifird, "Reputation and e-commerce: eBay auctions and the asymmetrical impact of positive and negative ratings," Journal of Management, vol. 27, pp. 279-295, 2001

[6] S. Schmidt, R. Steele, and T. Dillon, "DECo Arch: Trust and Reputation Aware Service Brokering in Digital Ecosystems " submitted to Inaugural IEEE International Digital Ecosystems and Technologies Conference. Cairns, Australia.

[7] S. Schmidt, R. Steele, and T. Dillon, "Trust and Reputation Ontologies for Electronic Business," submitted to International Conference on Enterprise Information Systems. Funchal, Portugal, 2007.

[8] L. A Zadeh, "Fuzzy Logic," Computer, vol. 21, pp. 83-93, 1988.

[9] E. H. Mamdani and S. Assilian, "An experiment in linguistic synthesis with a fuzzy logic controller," International Journal of HumanComputer Studies, vol. 51, pp. 135-147, 1999.

[10] S. Schmidt, R. Steele, T. Dillon, and E. Chang, "Building a fuzzy trust network in unsupervised multi-agent environments," International Workshop on Web Semantics. Agia Napa, Cyprus, 2005, pp. 816-825.

[11] E. J. Chang, F. K. Hussain, and T. S. Dillon, "Fuzzy nature of trust and dynamic trust modeling in service oriented environments," Proceedings of the 2005 workshop on Secure web services, pp. 75-83, 2005.

[12] M. E. Schweitzer, J. C. Hershey, and E. T. Bradlow, "Promises and lies: Restoring violated trust," Organizational Behavior and Human Decision Processes, vol. 101, pp. 1-19, 2006.

[13] S. Schmidt, R. Steele, T. Dillon, and E. Chang, "Applying a fuzzy trust model to E-commerce systems," Joint Conference on Artificial Intelligence. Sydney, Australia, 2005, pp. 318-329.

[14] X. Gu and Q. Zhu, "Fuzzy multi-attribute decision-making method based on eigenvector of fuzzy attribute evaluation space," Decision Support Systems, vol. 41, pp. 400-410, 2006.

[15] E. W. T. Ngai and F. K. T. Wat, "Fuzzy decision support system for risk analysis in e-commerce development," Decision Support Systems, vol. 40 , pp. 235-255, 2005.

[16] G. J. Klir and B. Yuan, Fuzzy sets and fuzzy logic: theory and applications: Prentice-Hall, Inc. Upper Saddle River, NJ, USA, 1994.

[17] C. Castelfranchi, R. Falcone, and G. Pezzulo, "Trust in information sources as a source for trust: a fuzzy approach," Proceedings of the second international joint conference on Autonomous agents and multiagent systems. Melbourne, Australia: ACM Press, 2003

[18] M. Lesani and S. Bagheri, Applying and Inferring Fuzzy Trust in Semantic Web Social Networks.

[19] B. Esfandiari and S. Chandrasekharan, "On How Agents Make Friends: Mechanisms for Trust Acquisition," 4 thWorkshop on Deception, Fraud and Trust in Agent Societies, 2001.

[20] L. Gong and S. M. Inc, "JXTA: A Network Programming Environment."

[21] M. Naor and U. Wieder, "A simple fault tolerant distributed hash table," in Second International Workshop on Peer-to-Peer Systems, 2003, pp. 88-97.

[22] E. Damiani, S. Paraboschi, P. Samarati, and F. Violante, "A reputationbased approach for choosing reliable resources in peer-to-peer networks," in Proceedings of the 9th ACM conference on Computer and communications security, 2002, pp. 207-216.

[23] S. Schmidt, R. Steele, and T. Dillon, "Towards Usage Policies for Fuzzy Inference Methodologies for Trust and QoS Assessment," International Conference on Computational Intelligence. Dortmund, Germany, 2006, pp. 263-274.

[24] E. Chang, F. Hussain, and T. S. Dillon, Trust and Reputation for Service-Oriented Environments: Technologies For Building Business Intelligence And Consumer Confidence: John Wiley \& Sons, 2005.

[25] E. H. Mamdani, "Advances in the linguistic synthesis of fuzzy controllers," International Journal of Man-Machine Studies, vol. 8, pp. 669-678, 1976

[26] The DEco Arch framework., http://exel.it.uts.edu.au/ $\sim$ sschmidt/decoarch 